\title{
De Cloporte en Janus : sur quelques représentations du portier dans la caricature française du XIX ${ }^{\mathrm{e}}$ siècle
}

Les caricatures de concierges, portiers et domestiques auxiliaires des portes et du passage dans les lieux publics de l'immeuble, ou privés de l'appartement, sont nombreuses au XIX ${ }^{e}$ siècle, en dessin comme en littérature. En effet, le concierge ou portier est un phénomène moderne à l'époque de Daumier et de Balzac.

Si l'incarnation féminine de la concierge a beaucoup été caricaturée sous forme visuelle et littéraire (voir Strobbe, 2013), l'homme-concierge a lui aussi fait l'objet de remarquables représentations caricaturales, que ce soit dans les Mystères de Paris d'Eugène Sue, les œuvres de Daumier, les journaux et les revues. Sa situation sociale, de même que celle de son local, est celle de l'entre-deux. Sa fonction de contrôle se heurte dès lors à la fois aux pressions du propriétaire de l'immeuble et à la résistance de ses locataires. Nous verrons la place spéciale du portier ou concierge dans la représentation caricaturale du XIX ${ }^{\mathrm{e}}$ siècle. Entre servilité et volonté de surpuissance, il fait l'objet de nombreux tiraillements. En effet, comment donc le concierge peut-il jongler entre les volontés conflictuelles du propriétaire et des locataires, sans trop souffrir dans son niveau de vie, ou trop perdre de sa dignité ? Il s'agit d'un défi difficile à surmonter, en raison de sa fonction même, et des attaques dont il est la cible facile et constante dans la littérature, mais aussi dans la société - que ce soit sous forme d'agressions ou encore de caricatures.

Le rôle du concierge évolue au fil des siècles - et son nom même se métamorphose suivant les représentations positives, négatives, et en tout cas souvent caricaturales, qui en sont faites.

Caroline Strobbe - Professeure Associée, The Citadel. Adresse de correspondence : The Citadel, Department of Modern Languages, Literatures and Cultures, 171 Moultrie Street, Charleston, SC 29409, U.S.A ; e-mail : cstrobbe@citadel.edu

ORCID iD : https://orcid.org/0000-0002-3909-373X 
Ainsi, dès le XVIII ${ }^{e}$ siècle, Louis-Sébastien Mercier dépeint le portier dans son Tableau de Paris (1783). Mercier emporte le lecteur dans sa marche parisienne, et y croque le portier, dans un clair-obscur textuel à la fois sérieux et humoristique, représentant une profession dont il dénonce acerbement la vanité.

La particularité de ce tableau brossé par Mercier est que ni propriétaires, ni locataires n'y figurent. Le portier - ou encore un Suisse ${ }^{1}$ - en émerge alors, comme figure d'opposition tant au lecteur qu'aux Parisiens, c'est-à-dire au public dans son ensemble ${ }^{2}$. Cet antagonisme agit comme un ressort de la caricature tout en dressant un portrait précis du Paris de la fin du XVIII siècle. L'une des origines du mot caricature a été retracée à l'italien caricare, qui signifie « charger». Et c'est en effet une charge que mène Mercier, dès le commencement de son chapitre sur les portiers : « Toute porte cochère a son portier bien ou mal soudoyé » (Mercier, 1783, p. 30).

La pièce à charge de Mercier se poursuit par une petite pointe contre le portier moderne - celui que nous reverrons également au XIX siècle - avant de sétendre sur l'origine des portiers - les Suisses - et leur physionomie quasi gargantuesque ; en effet, ceux-ci sont imposants, conséquence de leurs armes et de leur tournure physique :

Leurs têtes carrées \& leurs hallebardes pointues dominent la multitude. Celui qui essayerait de se glisser courrait risque dêtre comprimé \& étouffé entre deux masses helvétiques. J'ai vu un pauvre abbé mignon criant miséricorde, qu'il fallut dégager comme si l'éléphant de la ménagerie l’eut pressé contre la muraille. (Mercier, 1783, p.32)

La juxtaposition de ces formes massives, entre montagnes et murailles, de la zoologie et de ce pauvre abbé mignon (dont on rit aussi) se présente comme un outil efficace de la caricature littéraire.

À ce gigantisme des portiers suisses s'ajoute leur gloutonnerie : « Leur lourde physionomie ne paroît s'animer un peu qu'aux bals, lorsque le buffet est copieusement garni. Ils semblent tous porter sur leur front : nous n'aimons qu’à boire » (Mercier, 1783, p. 31).

Ce caractère grappilleur des portiers leur sera toujours attribué au XIX siècle, mais les caricaturistes cesseront de les représenter telles des montagnes suisses au fur-et-à-mesure que leur rôle se démocratisera - ou du moins qu'ils descendront de leur noble ou monumental piédestal pour travailler non plus essentiellement dans

1. Dès la fin du XVIII ${ }^{e}$ siècle, les portiers - souvent de nationalité suisse - gardaient monuments, jardins, et hôtels particuliers.

2. Dans son Histoire de la caricature moderne, Champfleury indique que « La caricature est [...] le cri des citoyens. Ce que ceux-ci ne peuvent exprimer est traduit par des hommes dont la mission consiste à mettre en lumière les sentiments intimes du peuple " (Champfleury, 1835, p. VII). Si l'on peut opposer que le concierge fait lui aussi partie du peuple, il faut néanmoins convenir que son activité particulière le met en relation voire en opposition avec la plus grande partie du peuple, qui prendra plaisir à reconnaître ses traits dépeints en caricature. 
des hôtels particuliers, jardins ou monuments, mais surtout au plus près de la population parisienne dont la réorganisation spatiale et sociale est en cours à Paris. Les Suisses avec leurs hallebardes font place aux portiers français, sans armes, et dès lors moins impressionnants que leurs prédécesseurs.

La ville au XIX ${ }^{e}$ siècle évolue, sous la logique d'une volonté de contrôle de l'espace public sur les plans politiques, hygiéniques, et sociaux. Cette politique, commencée bien avant Haussmann, s'accélère de plus en plus, alors que Paris multiplie les immeubles de rapport ou à loyer ${ }^{3}$. Or, ces immeubles modernes incluent de plus en plus souvent un portier ou concierge. En effet, les loges de portiers ne commencent à être construites que pour les appartements bâtis après les années $1820^{4}$. Le rôle de ce concierge double celui de la porte, car il doit s'assurer de la sécurité de l'immeuble, par un contrôle constant des entrées et des sorties, pour le compte des locataires, mais surtout du propriétaire qui l'emploie. En plus de ce rôle, le concierge doit aussi assurer la propreté des locaux, et représenter le propriétaire en acceptant ou refusant d'éventuels locataires, en collectant les loyers et en notifiant leurs congés aux locataires mauvais payeurs. En ce sens, il a un rôle de police privée qui va souvent le placer en porte-à-faux avec les intérêts des habitants de l'immeuble.

Daumier se plaît à représenter les relations entre concierges, locataires et propriétaires dans sa série "Locataires et Propriétaires " publiée dans Le Charivari. L’artiste se moque ainsi de la prétendue servilité du concierge face au propriétaire. Dans sa caricature Mossieu le propriétaire !... (fig.1) par exemple, il le représente, tel un soldat de pantomime, au garde-à-vous devant le propriétaire. Celui-ci, en robe de chambre et collants, adopte une attitude hautaine, comme en témoigne sa démarche élancée, pied pointé et buste cambré, circulant devant le portier au garde-à-vous, bras le long du corps, chapeau dans une main et arme - balai - dans l'autre, et devant le locataire qui le salue en baissant la tête, chapeau également à la main.

Daumier publie ensuite le Renouvelé de Gessler dans sa série Locataires et Propriétaires, et représente une nouvelle fois propriétaire et portier. Les traits sont différents, mais le titre semble faire écho à sa série de l'Histoire Ancienne. En effet, tandis que l'Histoire Ancienne représente des thèmes antiques à la statuaire vivifiée et modernisée, ce "renouvelé » fait référence au légendaire Gessler. Gessler était en effet un bailli suisse du XIVe siècle, qui aurait fait ériger son chapeau au bout d'une perche, afin de le faire saluer par tous les passants. Il aurait ensuite été tué par Guillaume Tell. Dans ce "Renouvelé », deux autres portier et propriétaire s'entourent encore d'un manteau d'autorité, alors que le portier dénonce au propriétaire celui des loca-

3. Nous adoptons ici la terminologie de Sharon Marcus. Le terme «maison de rapport» se rapporte à l'immeuble selon la perspective de son propriétaire, qui y voit un investissement et une source de revenus. Le potentiel économique de la «maison de rapport » est beaucoup plus important que celui de la «maison à loyer », qui indique simplement que l'appartement est disponible à la location (Marcus, 1999, p. 27).

4. Dans Apartment Stories, Marcus précise également qu'avant 1820, seuls les hôtels avaient des portiers (1999, p. 42). 
Figure 1.

Daumier, « Mossieu

le Propriétaire»,

Le Charivari,

10 mars 1854.

(c) www.daumier.org

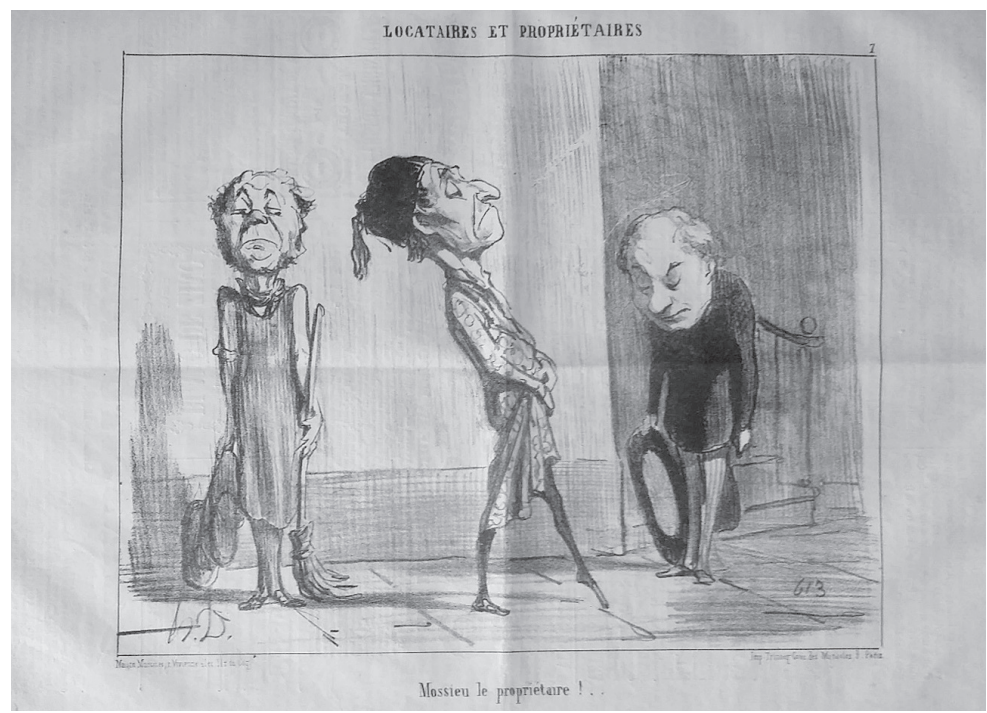

taires qui n’a pas salué son bonnet, planté sur un piquet dans la rue. L'attitude du portier dont le bras et le doigt sont tendus vers le chapeau ressemble à l'attitude d'un enfant qui rapporterait l'un de ses camarades. La réponse du propriétaire ne se fait pas attendre, et, tel un magistrat-Gessler, il énonce la sentence du tac-au-tac :

Le portier. - Mossieu il y a le locataire du cinquième qui s'est obstiné à passer aujourd'hui trois fois devant votre bonnet sans lui retirer son chapeau.

Le propriétaire. - Qu'on l'augmente de trois cents francs et en cas de récidive qu'il soit expulsé immédiatement de ma maison et que tous ses meubles soient confisqués ! (fig. 2)

Figure 2.

Daumier,

«Renouvelé

de Gessler »,

Le Charivari,

13 mars 1854.

(c) www.daumier.org

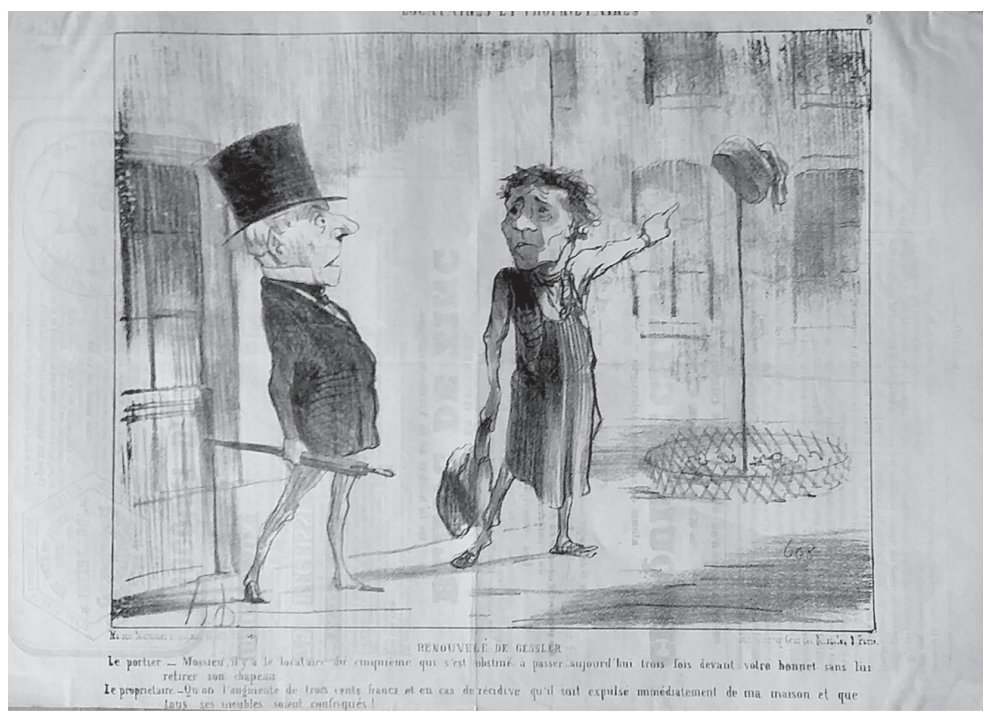


Notons l'arbitraire de la sentence énoncée par le propriétaire, son vocabulaire d'ordre légal -récidive, expulsé, confisqués -, qui crée un contraste humoristique dans l'enchevêtrement des fonctions d'un Propriétaire-Juge. L'arbitraire de cette sentence repose aussi sur le fait que de nombreux loyers avaient en fait des contrats oraux, et qu'il est donc possible de modifier avec célérité. Cette caricature s'enrichit aussi de l'opposition bonnet du propriétaire / chapeau du locataire, avec le locataire possédant le chef le plus formel, un chapeau étant a priori plus habillé qu'un bonnet. Le fier propriétaire passe enfin avec un chapeau haut-de-forme sur la tête, ce qui lui confère l'attribut du bourgeois. Devrait-il alors se méfier de la révolte du locataire-peuple, alors même que le bonnet juché sur une pique est aussi symbole révolutionnaire?

Le portier travaillant pour le propriétaire, il est aussi responsable de trouver des locataires pour chaque appartement. Alors que le portier est généralement et officiellement le membre du couple embauché pour remplir la fonction de concierge, le couple est néanmoins considéré comme entité de travail par le propriétaire qui les embauche. Si la portière est souvent représentée faisant faire la visite des appartements, le choix des locataires revient aux portiers mâles. Les femmes sont en effet incapables juridiquement, à moins dêtre veuves. C'est aussi seulement le portier qui est représenté prenant directement les ordres du propriétaire, ce qui peut être dû au fait que lorsqu'un couple est embauché, seul l'homme a la fonction officielle de portier.

Daumier représente ainsi Monsieur Vautour - nom archétypique du propriétaire dans ses caricatures - avec son nez crochu, et le doigt levé pour mieux donner ses ordres, en train de discuter avec son concierge dans une caricature publiée dans le Charivari en 1856. Encore une fois, le concierge l'écoute, chapeau bas :

$\mathrm{M}^{\mathrm{r}}$ Vautour : - Je ne saurai trop vous réitérer que je ne veux dans ma maison, ni enfants, ni chiens, ni serins..... et si une de mes locataires devenait enceinte..., je vous ordonne de lui signifier congé avant le terme..... j’ai remarqué qu'une des principales envies des femmes grosses, consiste à vouloir qu'on diminue leurs loyers !..... (Daumier, 20 octobre 1856, DR 2839)

Le ton du propriétaire est autoritaire, et l'humour de cette légende provient de l'accumulation des êtres que refuse le maitre des lieux, les enfants étant au même rang que les animaux, aussi insignifiants peut-être que des serins. Le jeu de mots axé autour de "terme ", entre le terme de la grossesse et le terme du loyer, fera aussi rire le public du Charivari, aux dépens de ce propriétaire.

L’attitude presque prostrée du portier est à mettre en parallèle avec son comportement dédaigneux lorsqu'il est à même de refuser une location à une mère de famille. Le travail caricatural se fait alors non seulement par l'impact qu'a en soi une caricature, mais aussi par la comparaison entre elles. Ces lithographies se complètent en effet autour de la discrimination envers les femmes mères. Ainsi, en juillet 1856, Le Charivari publie une caricature de Daumier représentant une femme et son enfant dans l'ombre, devant la loge du concierge, tandis qu'il est dans la lumière au pre- 
Figure 3.

Daumier, «C'est inutile que je vous

fasse voir mon appartement...», Le Charivari, 30 juillet 1856. (c) www.daumier.org

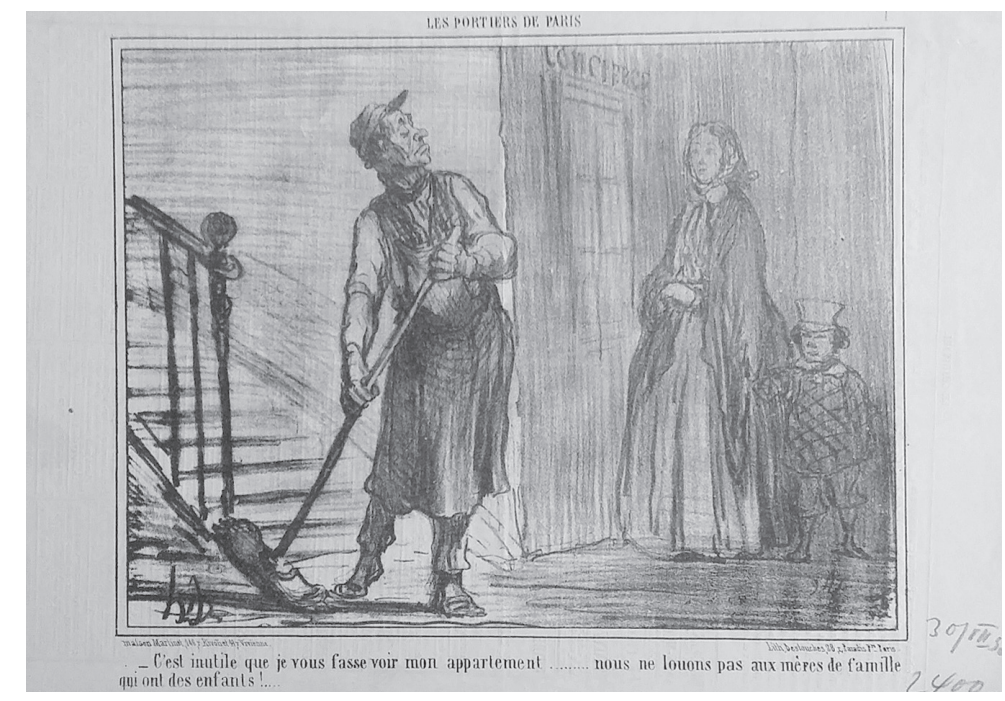

mier plan, en train de balayer les escaliers. Le nez en l'air et les yeux fermés, ce qui lui donne l'air dédaigneux, il claironne : "C'est inutile que je vous fasse voir mon appartement..... nous ne louons pas aux mères de famille qui ont des enfants !... » (fig. 3). Ce ton emphatique associé à l'insistance du portier sur le refus des mères qui - nécessairement - ont des enfants ajoute une couche d'ironie à cette caricature. Le public peut à son tour dédaigner ce portier hautain mais obtus, qui pourtant joue un si grand rôle dans la vie des Parisiens.

Les locataires ont en effet de fréquents contacts avec leurs concierges, qui sont les hommes de confiance des propriétaires. Le portier est - ainsi que son nom l'indique - préposé à la porte. Il doit la garder, c'est-à-dire être prêt à l'ouvrir ou à la fermer à n'importe quelle heure du jour, voire de la nuit. Cet attachement à la porte ainsi que son rôle font l'objet de caricatures mordantes, telle que celle publiée par Le Charivari le 9 mars 1838. Cette lithographie titrée "Le boul-dogue (chien de garde) ", porte le numéro 10 de la série Cours d'Histoire Naturelle (fig.4). Ainsi que son titre et son affiliation à l'histoire naturelle le laissent supposer, la planche décrit le comportement du chien de garde, vu dans son habitat naturel - c'est-dire à la porte des immeubles dont il a la charge. Dessin et texte s'illustrent l'un l'autre, tandis qu'un concierge au visage canin, la bouche ou la gueule ouverte, aboie sur une dame et son fils. Même si le bouledogue est retenu par une demi-porte où il appuie ses mains, on le voit prêt à s'élancer. La dame met la main sur son cœur dans un geste exprimant à la fois sa peur et sa surprise, son fils se penchant en arrière pour mieux regarder le portier. Cette caricature graphique à elle seule suffirait pour faire rire de l'attitude rébarbative reprochée aux concierges, et elle correspond bien au rire qui, selon Baudelaire, « vient de l'idée de sa propre supériorité » (Baudelaire, 1976, p. 530). Le texte ajoute cependant une forte pointe : 


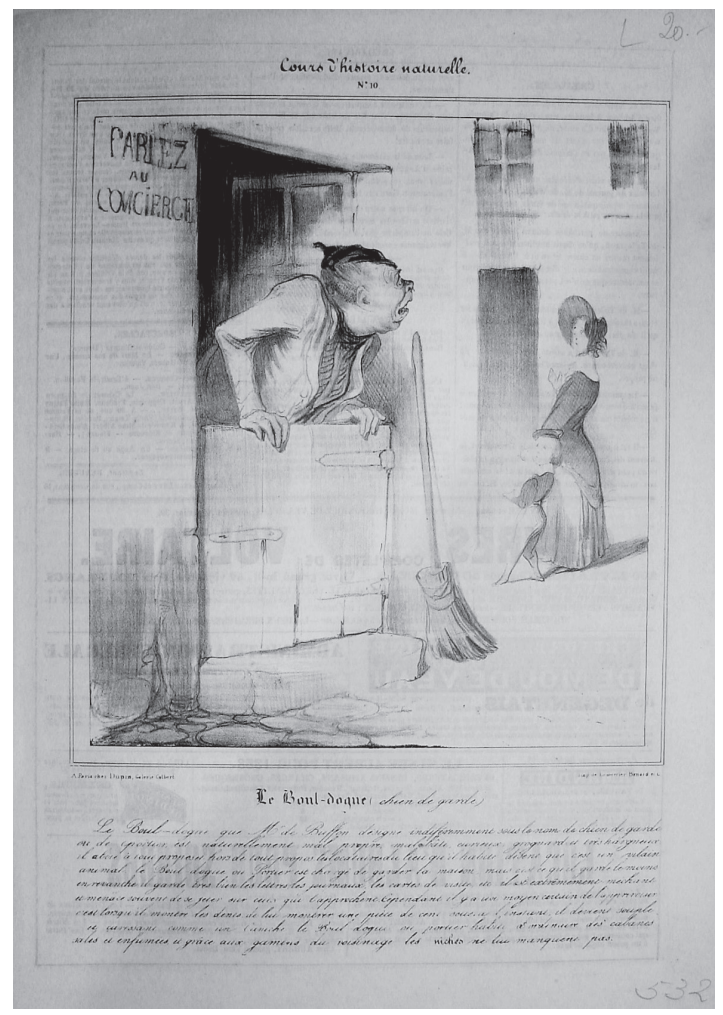

Figure 4. Daumier, « Le boul-dogue (chien de garde) », Le Charivari, 9 mars 1838. @ www.daumier.org
Le Boul-dogue que Mr. de Buffon désigne indiféremment [sic] sous le nom de chien de garde ou de portier est naturellement mal propre, mal bâti, curieux, grognard, et très hargneux. il aboie à tout propos et hors de tout propos. les locataires du lieu qu'il habite disent que c'est un vilain animal. le Boul-dogue ou Portier est chargé de garder la maison, mais c'est ce qu'il garde le moins. en revanche il garde très bien les lettres, les journaux, les cartes de visite, etc. il est extrêmement méchant et menace souvent de se jeter sur ceux qui l'approchent. Cependant, il y a un moyen certain de l'apprivoiser c'est, lorsqu'il montre les dents, de lui montrer une pièce de cent sous, à l'instant, il devient souple et caressant comme un Caniche. le Boul-dogue ou portier habite d'ordinaire des cabanes sales et enfumées, et grâce aux gamins du voisinage les niches ne lui manquent pas.

Le champ lexical met l'accent sur le caractère hargneux ou intéressé du portier, et en tout état de cause, sur son animalité. La caricature par animalisation du sujet est fréquente au XIX ${ }^{\mathrm{e}}$ siècle, siècle des catégorisations et des physiologies. En 1841, Honoré Daumier participe ainsi au Museum Parisien, de Louis Huart, entreprise éditoriale dont le sous-titre à longueur encyclopédique ancre la population parisienne en particulier dans différents types de catégories : Histoire physiologique, pittoresque, philosophique et grotesque de toutes les bêtes curieuses de Paris et de la Banlieue pour faire suite à toutes les éditions des Euvres de Buffon. Huart intitule son chapitre sur les concierges « Le cloporte» :

Depuis peu de temps seulement cette dénomination est en circulation dans la société, mais elle nous semble devoir être promptement adoptée par tous les véritables amateurs d'histoire naturelle parisienne. Le Cloporte est devenu le synonyme de portier, et ce nom est d'autant plus juste que ce fonctionnaire privé a pour mission spéciale de se tenir constamment recoquevillé [sic] dans une petite fente du logis, à l'instar du coléoptère nommé plus haut. Pour seconde mission non moins spéciale, il a le droit de veiller 
constamment à ce que la porte soit close. Vous voyez donc que l'antique concierge, suisse ou portier, a deux fois le droit de s'appeler Cloporte. (Huart, 1841, p. 81-82)

Néologisme et calembour, cette désignation de « cloporte » pour le portier devient populaire, et entre dans la langue courante. Pierre Sudant et Jean-Marc Stébé remarquent ainsi qu'en "1850, le mot Cloporte est couramment utilisé, au féminin et dans un sens péjoratif, pour désigner la concierge » (Sudant, 2002, p. 25).

Retenons ici que la caricature, qui veut rire et faire rire du portier, s'appuie néanmoins sur des éléments réalistes, car de nombreuses loges de portiers étaient exigües et insalubres. L'une des plus célèbres en littérature est sans doute celle des concierges Pipelet, dont le nom est devenu synonyme de bavards incontinents. Dans les Mystères de Paris, Eugène Sue décrit l'accès à cette loge :

Une allée sombre, étroite, conduisait à une petite cour ou plutôt à une espèce de puits carré de cinq ou six pieds de large, complètement privé d'air, de lumière ; réceptacle infect de toutes les immondices de la maison, qui y pleuvaient des étages supérieurs, car des lucarnes sans vitres s'ouvraient au-dessus du plomb de chaque palier.

Au pied d'un escalier humide et noir, une lueur rougeâtre annonçait la loge du portier ; loge enfumée par la combustion d'une lampe, nécessaire même en plein jour pour éclairer cet antre obscur [...]. (Sue, 1851, p. 52)

Il ne s'agit pas ici d'une caricature, mais d'une description tout à fait réaliste, qui montre que le lien entre logement sombre et malsain et caricature du portier en cloporte est effectivement vite tracé 5 . Mais Sue ne se contente pas de ce portrait réaliste d'une loge de portier. Il représente aussi un M. Pipelet engourdi par les facéties de Cabrion. Il est tantôt représenté " seul dans sa loge, travailleur consciencieux et infatigable " s'occupant de "restaurer la botte qui lui était plus d’une fois tombée des mains lors de la dernière et audacieuse incartade de Cabrion" (1851, p. 185), tantôt représenté désespéré, et dans un effort de survie pathétique et comique, allant au commissariat pour dénoncer les actions de Cabrion, le rapin farceur. Alors que Cabrion a épinglé le portrait réaliste de sa propre tête au-dessus de la couche nuptiale des Pipelet, Alfred Pipelet se refuse littéralement, et avec sérieux, à lui faire tomber la tête :

« Non, reprit Alfred, je n'aime pas le sang, mais j’ai le droit de réclamer la réclusion perpétuelle de cet être malfaisant ; mon repos l'exige, ma santé me le commande... la loi

5. Deaucourt atteste qu'un «sondage effectué sur les plans du début du siècle donne avec une superficie moyenne de $15 \mathrm{~m}^{2}$ une image des conditions de logement [...]. De fortes différences existent d'une rue à l'autre $\left(20 \mathrm{~m}^{2}\right.$ rue de Rivoli contre $8 \mathrm{~m}^{2}$ Quai des Ormes), d'une maison à l'autre : par le jeu des pièces doubles et des soupentes, certaines loges s'étendent jusqu'à $30 \mathrm{~m}^{2}$ quand d'autres tiennent dans $5 \mathrm{~m}^{2} »$ (Deaucourt, 1992, p. 74). 
doit m’accorder cette réparation... sinon je quitte la France... ma belle France ! Voilà ce qu'on y gagnera. »

Et Alfred, abîmé dans sa douleur, sortit majestueusement de sa loge, comme une de ces imposantes victimes de la fatalité antique. (1851, p. 188)

Les traits caricaturaux proviennent ici du contraste entre le châtiment que souhaite le pauvre concierge à l'encontre de Cabrion et l'humanité qu'il désire conserver. De même, l'opposition entre le malheureux portier et des figures éventuellement mythologiques de la « fatalité antique » ajoute encore à la pointe dirigée contre le concierge. Cependant, si l'on peut ici rire de lui, le rire est aussi empreint de pitié pour la misérable victime de Cabrion. Ce ne sera plus le cas lorsque le personnage de Cabrion sera repris par la presse. Le journal L'Anti-Concierge, qui suggère d'essuyer ses pieds « quand ils sont très sales sur la tête de votre concierge " fait aussi la réclame pour le «Conciergicide Cabrion. 1000fr le flacon. N'est distribué qu'aux personnes justifiant de leur qualité de locataires. Dépôt à l'Anti-Concierge » (Jouy \& Sapeck, 1881, p. 4) ${ }^{6}$.

Le portier, tombé d'homme à animal en caricature, est aussi élevé en dieu. Ces techniques d'amenuisement ou au contraire d'élévation spectaculaire participent de l'effet d'instantanéité propre à nombre de caricatures. Daumier publie son PIPELET RÉPÉTANT son SOURIRE de DÉCEMBRE : " Prenons garde ! prenons garde ! / Car le jour de l'An nous regarde » (fig. 5).

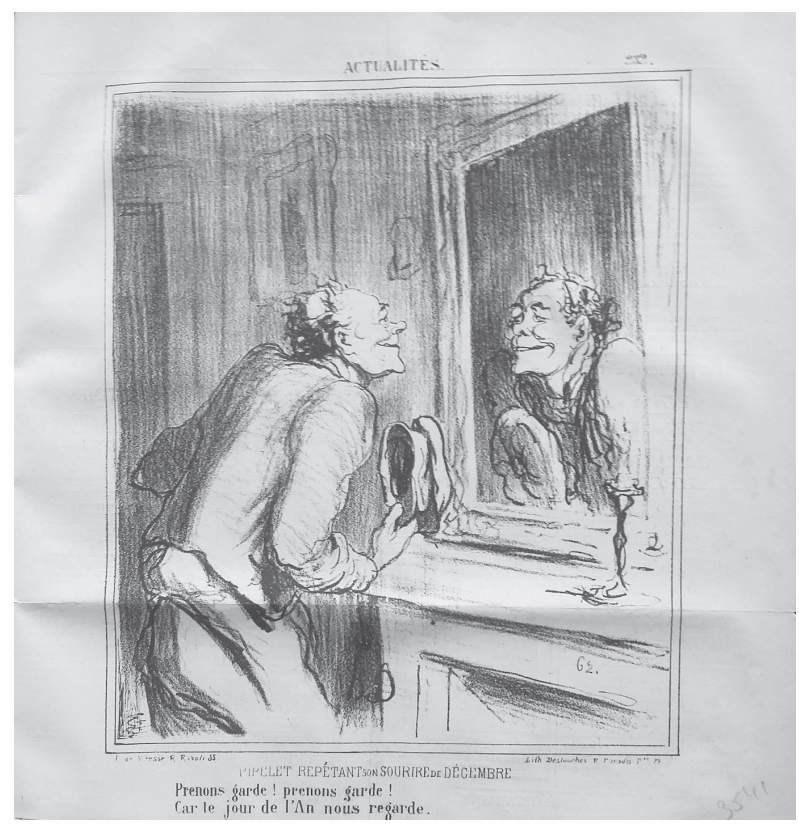

Figure 5. Daumier, «PIPELET RÉPÉTANT SON SOURIRE de DÉCEMBRE », Le Charivari, 13 décembre 1866 .

(c) www.daumier.org

6. L'Anti-Concierge est également mentionné dans l'article de Strobbe dans Romantisme (2013, p. 136). 
Tandis que le Pipelet de Daumier s'entraine à sourire devant son miroir, le portier se révèle en Janus dans la caricature Les 2 Faces de $M^{r}$ Janus de Linton pour Le Monde Illustré (fig. 6). Dans ce dessin, l'artiste dessine un portier à deux visages, dont l'un se penche et sourit obséquieusement à son locataire, tandis que l'autre est hautain, bouche et yeux fermés. Le concierge est représenté avec ses attributs : les clefs, accrochées au mur, ainsi que le balai sous le bras et le courrier en main, dans une loge exigüe et peuplée d'un chat et d'un chien. On remarque que le locataire en pardessus et chapeau haut-de-forme est à moitié entré dans la loge, et, s'appuyant sur la demi-porte, il semble être le maître tandis que le concierge serait le serviteur. La représentation du portier en Janus est particulièrement bien choisie, et ce à plusieurs niveaux. En effet, selon Gérard Capdeville, Janus est « Dieu des portes, réelles ou symboliques, qu'il ouvre et ferme » (1973, p. 416). Il est aussi « Dieu des passages et des commencements, [...] tout naturellement chargé de la protection du début de l'année [...] mais aussi du début de chaque mois » (p. 424), ce qui accentue encore la pointe caricaturale : ce dessin fait en effet la couverture du journal, en ce premier janvier 1859. Les locataires comprendront donc l'intérêt de donner de bonnes étrennes à ce dieu qu'ils ne peuvent esquiver, auréolé d'une pièce de trois francs, sous l'inscription : "O ! St Jour de l'An ». L'article de Fulgence Girard, qui accompagne cette caricature, remarque : "N’est-il [Janus] pas dès lors, dans sa traduction la plus pittoresque et la plus saisissante, la physiologie du portier de la Saint-Sylvestre... du trente et un décembre au premier janvier» $(1859$, p. 1$)$.

Si le portier est Janus au sourire temporaire pour le locataire, sa duplicité est néanmoins ancrée dans les esprits de ses contemporains : servile face à un propriétaire autoritaire et arbitraire, il règne sur son immeuble avec une autorité sans cesse contestée, à la fois dieu des passages, chien ou cloporte. Sa présence constante et la quasi-impossibilité de lui échapper forment un ciment qui lie de nombreuses

Figure 6. Linton, « Les 2 Faces de Mr. Janus », Le Monde Illustré, 90, 1 Janvier 1859. Collection de l'auteure.

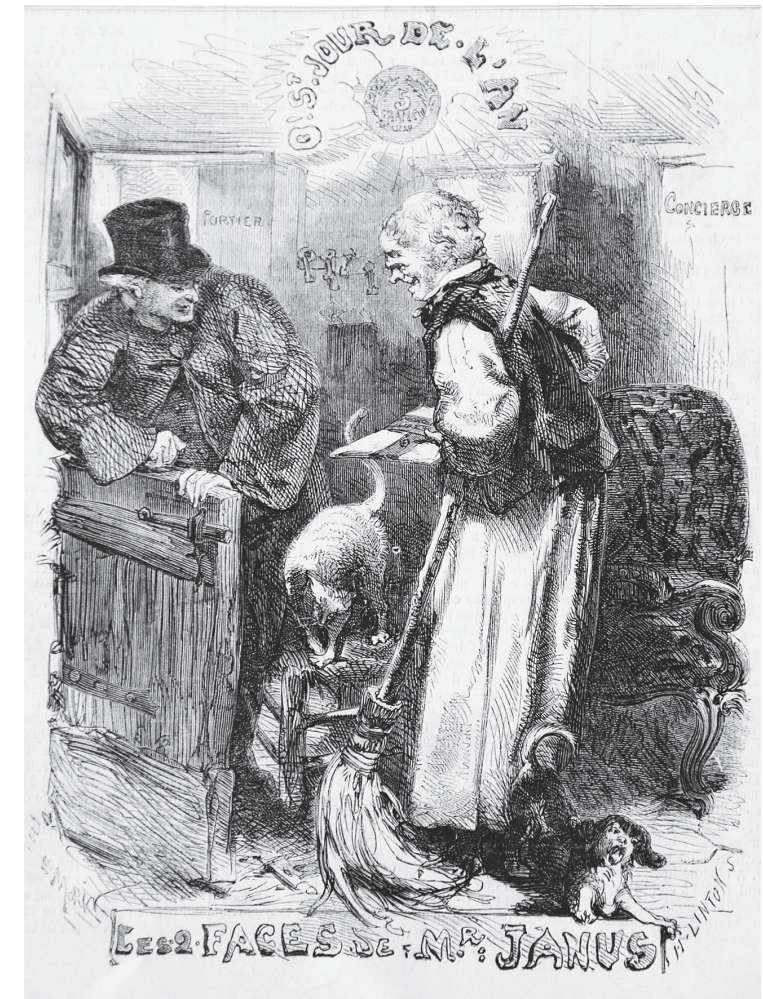


classes sociales, qu'il s'agisse de propriétaires, de locataires, hommes ou femmes, d'habitants de Paris et de maints lecteurs de la presse. Caricaturiste et lecteur/spectateur y partagent une culture commune, leur permettant de prendre une légitime revanche sur un personnage si essentiel à leur vie. C'est sans doute dans la nécessité de sa fonction et dans son autorité que réside, pour le portier, la compensation de toutes les moqueries dont il a pu faire l'objet.

\section{RÉFÉRENCES}

Baudelaire, Ch. (1976). De l'Essence du Rire et généralement du comique dans les arts plastiques. Euvres Complètes. T. 2. C. Pichois (éd.). (p. 525-543). Paris : Gallimard.

Capdeville, G. (1973). Les épithètes cultuelles de Janus. Mélanges de l'École française de Rome. Antiquité 85(2), 395-436.

Champfleury. (1835). Histoire de la caricature moderne. Paris : Dentu.

Deaucourt, J. (1992). Premières loges : Paris et ses concierges au XIX'e siècle. Paris : Éditions Aubier.

Girard, F. (1859). Monsieur Janus. Le Monde Illustré 90(1), 1.

Huart, L. et Daumier, H. (1841). Muséum Parisien. Histoire physiologique, pittoresque, philosophique et grotesque de toutes les bêtes curieuses de Paris et de la Banlieue pour faire suite à toutes les éditions des Euvres de Buffon. Paris : Beauger et Cie.

Lemesle, H. (2003). Vautours, Singes et Cloportes: Ledru-Rollin, ses locataires et ses concierges au XIXe siècle. ADHE.

Marcus, S. (1999). Apartment stories: City and Home in Nineteenth century Paris and London. Berkeley: University of California Press.

Mercier, L.-S. (1783). Tableau de Paris. Hambourg : Virchaux \& Compagnie.

Stébé, J.-M. et Sudant, P. (2002). Gardiens d'immeubles au coeur de la ville : figures, métamorphoses et représentations. Bruxelles : De Bœck.

Strobbe, C. (2013). Concierge : la difficile (recon)naissance de la femme. Romantisme 159(1), 135-146.

Sue, E. (1851). Les Mystères de Paris. Paris: Schneider.

Sapeck (pseud. de Bataille) et Jouy, J. (1881) L'Anti-Concierge. Organe officiel de la défense des locataires, $\mathrm{N}^{0} 1$. Déc 1881.

\section{FIGURES}

Daumier, H. (10 mars 1854). Mossieu le Propriétaire. Le Charivari. DR 2576. Daumier Register. http://www.daumier-register.org/werkview.php?key=2576

Daumier, H. (13 mars 1854). Renouvelé de Gessler. Le Charivari. DR 2577. Daumier Register. http://www.daumier-register.org/werkview.php?key=2577

Daumier, H. (30 juillet 1856). C'est inutile que je vous fasse voir mon appartement..... Le Charivari. DR 2825. Daumier Register.

http://www.daumier-register.org/werkview.php?key=2825 
Daumier, H. (9 mars1838). Le boul-dogue (chien de garde). Le Charivari. DR 532. Daumier Register. http://www.daumier-register.org/werkview.php?key=532

Daumier, H. (20 octobre 1856). Mr. VAUTOUR : - Je ne saurais trop vous réitérer [...] Le Charivari. DR 2839. Daumier Register.

http://www.daumier-register.org/werkview.php?key=2839

Daumier, H. (13 décembre 1866). PIPELET RÉPÉTANT son SOURIRE de DÉCEMBRE.

Le Charivari. DR 3541. Daumier Register.

http://www.daumier-register.org/werkview.php?key=3541

Linton, H. (1 janvier 1859). Les 2 Faces de Mr. Janus. Le Monde Illustré, 90.

RÉSUMÉ : Les caricatures de concierges, portiers et domestiques auxiliaires des portes et du passage dans les lieux publics de l'immeuble, ou privés de l'appartement, sont nombreuses au XIX ${ }^{\mathrm{e}}$ siècle, en dessin et en littérature. En effet le concierge ou portier est alors un phénomène moderne, et sa situation sociale, de même que celle de son local, est située dans l'entre-deux. Sa fonction de contrôle se heurte dès lors à la fois aux pressions du propriétaire de l'immeuble et à la résistance de ses locataires. Le portier ou concierge occupe donc une place spéciale dans la représentation caricaturale du XIX ${ }^{\mathrm{e}}$ siècle. Entre servilité et volonté de surpuissance, il fait l'objet de nombreux tiraillements. Alors qu'il est écartelé socialement entre locataire et propriétaire, ses représentations caricaturales s'étirent entre animal et dieu.

Mots-clés : caricature, portier, Sue, Daumier, Le Charivari

\section{From Cloporte to Janus: about some representations of doormen in the 19th-century French caricatures}

ABSTRACT: There are numerous caricatures of male concierges or doormen in the nineteenth-century texts and drawings. They constitute a very modern phenomenon, as new building layouts of the time, or new buildings in general, almost always included a lodge. Their role of controlling the whereabouts of people getting in and out of the building was subject to the contradictory pressures from landlords and tenants alike. We will try to demonstrate how the doormen and concierges belonged to the in-between places in the nineteenth-century French caricature, with their social position stretched between their landlords and tenants, a function that allowed them to combine servility and an excessive show of strength, and resulted in their depictions ranging from animals to gods.

Keywords: caricature, doorman, Sue, Daumier, Le Charivari 